\title{
Surfing, spinning, or diving from reef to reef: effects on population connectivity
}

\author{
Claire B. Paris*, Laurent M. Chérubin, Robert K. Cowen
}

Rosenstiel School of Marine and Atmospheric Science, University of Miami, Miami, Florida 33149-1098, USA

\begin{abstract}
Coral reef fish have considerable larval behavioral capabilities that can lead to successful completion of the early pelagic life phase. In particular, vertical migration during ontogeny increases retention near natal reefs and decreases losses due to transport by currents. For those larvae that are not returning home, the relative influence of behavior (biology) and currents (physics) on their arrival pattern among adjacent and distant reefs is not known. Moreover, interactions of the naturally small-scale larval movements with those of larger-scale currents need to be evaluated with regard to the spatial patterns of recruitment. We used an offline Lagrangian stochastic modeling approach to explore the relative influence of physical (i.e. eddy perturbation, diffusion) and biological processes (i.e. vertical movement, mortality) on the connectivity of the coral reef fish population in the western Caribbean, a region with complex geomorphology and circulation. This study revealed that the impact of larval behavior extends beyond enhancing the process of self-recruitment by changing population connectivity patterns. Connectivity was significantly influenced by larval vertical movement, survival, and by the eddy field, all controlling arrival patterns near reefs. A sensitivity analysis was done to gauge the robustness of the results by varying the model parameters. We found that particle-tracking models with homogeneous parameterization of the sub-grid motion tended to bias dispersal from and along the reef track, which can be mitigated by using spatially explicit parameters calculated from the Eulerian velocity fields. Finally, larval survival emerged as a key component for connectivity estimates, the study of which poses a great challenge in tropical ecosystems.
\end{abstract}

KEY WORDS: Modeling fish larvae $\cdot$ Transition probability matrix $\cdot$ Dispersal kernel · IBM · Population connectivity $\cdot$ Lagrangian $\cdot$ Stochastic model $\cdot$ Spin $\cdot$ Offline model

\section{INTRODUCTION}

The distance and direction of larval dispersal have considerable influence on the demography and genetic structure of marine species. Recent studies indicate that despite their small size, coral reef fish larvae are not passive (for a review, see Leis 2007, this Theme Section [TS]). They have a diversity of traits and considerable behavioral capabilities that can lead to successful completion of the early pelagic life phase (Fig. 1). In particular, vertical migration during ontogeny increases retention near natal reefs and decreases dispersion losses, likely enhancing survival (Paris \& Cowen 2004). Larvae can come back to their native reefs or can be exchanged among breeding sub- populations. However, the dynamics of these interactions at both the individual and population levels are not fully understood (deYoung et al. 2004). For those larvae that do not return home, the extent to which their behavior influences their arrival pattern among adjacent and distant reefs (or larval connectivity network) is not known. More importantly, interactions of the small-scale larval movements with transport processes due to larger-scale currents need to be quantified with regard to the spatial patterns of recruitment. Since actively moving larvae may be diluted and to some extent dispersed by currents, an in situ study of them is very difficult. Spatially explicit, individualbased modeling (IBM) has emerged as a key tool for understanding organism-environment interactions 


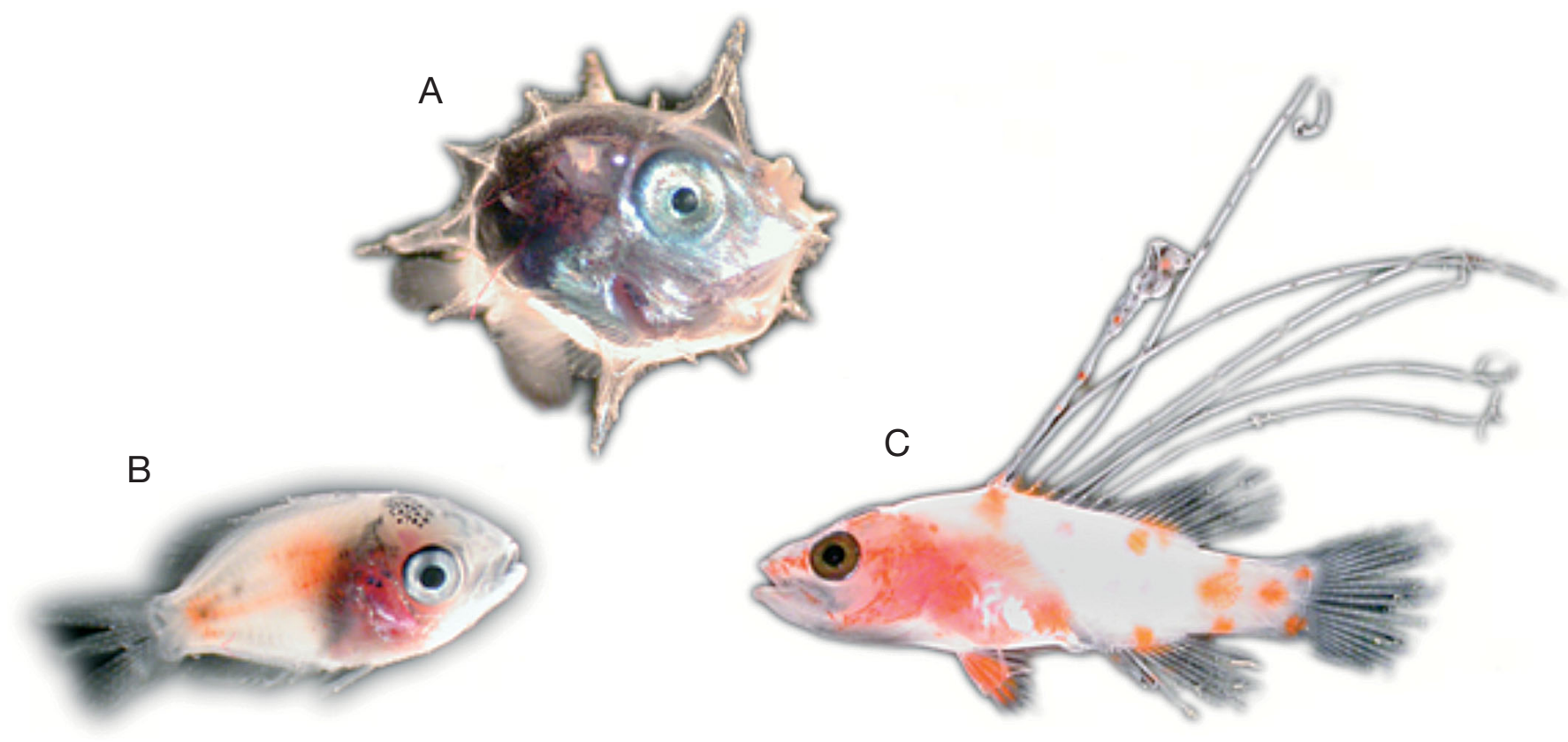

Fig. 1. Morphological diversity in fish larvae: (A) the pelagic sunfish Mola mola develops heavy pigmentation (melanophores) presumably to sustain UV radiations near the ocean surface, while coral reef fish larvae such as (B) the bicolor damselfish Stegastes partitus and (C) the candy bass Liopropoma sp. are mostly clear, with red-orange pigments (erythophores); as the ocean strongly absorbs colors in the red-orange part of the spectrum, these erythophores would appear dark at the depth where these larvae were captured (20 to $40 \mathrm{~m}$ and 60 to $80 \mathrm{~m}$ strata, respectively; Paris \& Cowen 2004, R. K. Cowen unpubl. data), breaking up the larva's shape. Diversity in shape and presence of appendages and elongated spines imply that these larvae occupy specific behavioral niches in the water column

(Werner et al. 2001) and is particularly relevant to investigate larval fish fluxes in the complex coral reef ecosystem. Currently, the spatial scales of the dispersal of reef fish larvae are estimated using indirect and empirical techniques (Thorrold et al. 2002, Jones et al. 2005) or modeling approaches (Cowen et al. 2000, 2006, James et al. 2002). The interactions between physical and biological factors and their role in shaping populations have been previously discussed in landscape ecology (Levins 1969), but this discussion is a relatively recent development for marine ecosystems (Barber et al. 2002, Baums et al. 2006, Cowen et al. 2006). The spatial arrangements and connectivities of marine populations are poorly understood, yet they are assumed to enhance resilience to exploitation and be of critical importance for population persistence (Kinlan et al. 2005, Hasting \& Botsford 2006). Measures of natal dispersal are typically determined by the dispersal kernel $k(x, y)$, defined as the probability of a larva settling at a distance $x$ given that it was released at $y$. The modal dispersal distance from the dispersal kernel has demographic relevance (e.g. population spatial pattern, persistence), while the tail, representing longdistance dispersal, is relevant on an evolutionary level (e.g. genetic mixing, species persistence; Hanski \& Gaggiotti 2004, Steneck et al. 2006). Models of population connectivity should thus emphasize where and how frequently larval linkages occur, and how these observed patterns are created. Here, we use a biophysical model designed to output spatially explicit transition probability matrices (or connectivity matrices) from which dispersal kernels are generated. The connectivity matrix describes the probability that an individual moves during its pelagic larval stage from the birthplace (or source population) to its settlement location (or sink population) as a settling larva, all in a 3-dimensional dynamic system. Such transition probability matrices are of considerable value for metapopulation and genetic studies (Hedrick 2000), as well as for spatial management and conservation issues (Urban \& Keitt 2001). We show that they also provide a method to quantify the relative influence of biological and physical factors on realized larval dispersal and on levels and spatial patterns of recruits.

Lagrangian stochastic models (LSMs) have been developed for modeling atmospheric transport problems (e.g. Sawford 1999). These are being increasingly applied to track the dispersal of larvae (see Levin 2006 for a review). Motions at small scales that are not resolved by ocean general circulation models (OGCMs) are usually parameterized. These include motions due to small-scale currents and random or oriented motions of individual, simulated larvae (Paris et al. 2002, Codling et al. 2004). Parameterization of subgrid-scale processes is critical for accurate modeling of trajectories and capturing variability (Siegel et al. 2003, deYoung 
et al. 2004). Yet, to date, very few larval dispersal studies address the parameterization of the random component statistics in tracking models. The extent to which parameterization of the Lagrangian parameters and larval traits plays a role in estimating population connectivity, and influences arrival patterns among native, adjacent, and distant reefs, is not known. Moreover, even though larvae are irrefutably not passive (Leis 2006), the question still remains of how biological processes weigh against oceanographic process.

To answer these questions, we present a numerical experiment approach that isolates parameters from the larval tracking algorithm and quantifies the relative influence of physical and biological processes on the spatial scales and patterns of larval exchange. Our main goal was to test the hypothesis that the importance of larval traits in shaping dispersal and population networks is comparable with that of ocean circulation. The primary objectives were to (1) use various LSM configurations to isolate and determine the most sensitive physical parameters in estimating dispersal kernels for the study region, (2) investigate the interactions of those parameters with biological parameters reproducing early life-history traits of coral reef fish, and (3) rank the relative importance of parameters and their interactions in estimating population networks in the meso-American region.

\section{BIOPHYSICAL MODEL SETUP}

We simulated the dispersal and recruitment of larvae using a spatially explicit Lagrangian stochastic framework (e.g. Hermann et al. 2001), linking biological and physical modules or code units. The coupled biophysical IBM tracked individual larvae within a population, each interacting with the environment based on its present state and past history, and produced probabilistic simulations of both larval trajectories and connectivity matrices (Fig. 2). Integration of archived velocity fields of an ocean circulation model moved virtual larvae (particles). A stochastic scheme parameterized the subgrid turbulent motion, which was added to the particle displacement at each integration time step. A biological module simulated larval traits (i.e. mortality, ontogenetic vertical migration, pelagic duration) as a function of developmental stage and settlement habitat, while a seascape module tracked the presence of the particles in selected coral reef areas. Explicit treatment of spatial history was achieved by simultaneous inclusion of the seascape (e.g. spawning and settlement areas) and the velocity fields in the IBM.
Hydrodynamic module. The hydrodynamic data used was generated with the 3-dimensional Regional Ocean Modeling System (ROMS), which is discretized in coastline- and terrain-following curvilinear coordinates ( $\sigma$-coordinates model; Marchesiello et al. 2003, Shchepetkin \& McWilliams 2004). We used the UCLA ROMS version, which performs local refinement via nested grids (Adaptive Grid Refinement in Fortran; Blayo \& Debreu 1999) and has the ability to manage an arbitrary number of embedded levels as well as to do adaptive grid-refinement. The model has 25 vertical layers, and its state variables (temperature, salinity) at the open ocean boundaries are relaxed monthly to the Levitus ocean (http://ingrid.ldeo.columbia.edu/ SOURCES/.LEVITUS94/) climatology (World Ocean Atlas). Tides are set at the boundary by the TPXO6 global tide model (www.esr.org/polar_tide_models/ Model_TPXO62_load.html). Monthly varying surface
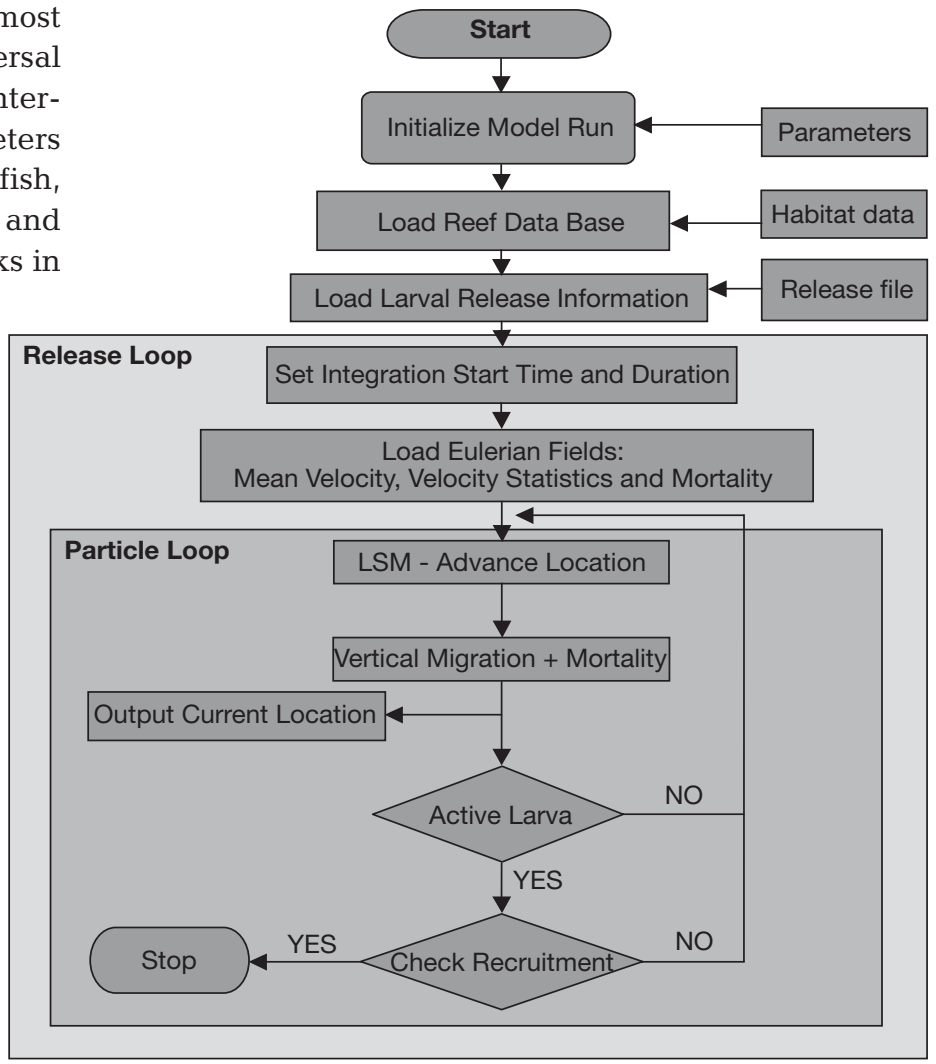

Fig. 2. Flowchart of the offline larval tracking system. The general algorithm for the code consists of several steps: (1) initialization reads the grid coordinate of the ocean model and spatially explicit habitat and population information, (2) individual particles are advanced using the ocean model output frequency, and (3) individual larval behavior and mortality rate is imposed. Finally, if a larva is competent, its location is checked to verify if it falls near settlement habitat. It is then assumed to have successfully recruited, and the time and day and location of recruitment are saved. Otherwise, the larval dispersion is continued until it is recruited or the model is integrated for the number of days specified by the maximum competency period 
fluxes (wind, rain, solar, radiative heat fluxes, evaporation) were obtained from the Comprehensive Ocean Atmosphere Dataset (http://icoads.noaa.gov/status.html) (COADS) climatology. Our region of simulation was the Meso-American Barrier Reef System (MBRS) in the western Caribbean, which encompasses one of the largest barrier reefs, off the coast of Belize (Fig. 3). This region is of interest to this study because of the presence of submesoscale eddies ( 10 to $100 \mathrm{~km}$ diameter), coastal currents, and topographic features that constrain the circulation and connectivity (Tang et al. 2006). In general, the modeled circulation was consistent with observations from float trajectories (Richardson 2005) and with model simulations from Ezer et al. (2005). On average, the Caribbean Current flows westward between 18 and $19^{\circ} \mathrm{N}$ and veers north along the
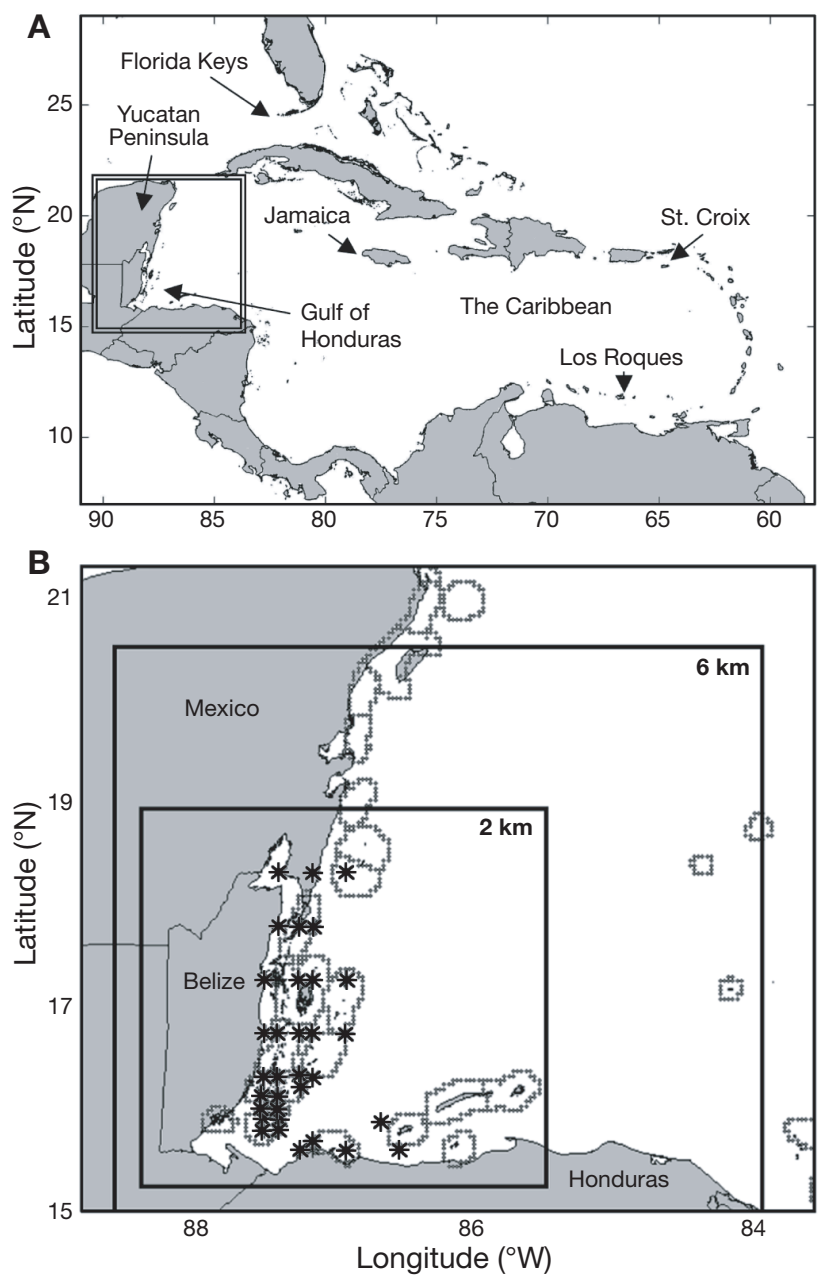

Fig. 3. (A) The Caribbean region with insert (enlarged in Panel B) showing the domain of the numerical experiments with the boundaries of the Regional Ocean Modeling System parent grid $(6 \mathrm{~km})$ and child grid $(2 \mathrm{~km})$. Asterisks indicate the release locations of simulated online floats $(n=70)$ used to calculate the intrinsic Lagrangian parameters; only those released within the reef habitat (gray lines) were used for analyses of larval dispersal $(n=48)$
Yucatan Peninsula into the Gulf of Mexico. South of the Caribbean Current, cyclonic eddies are formed and constrain the circulation in the Gulf of Honduras (Fig. 3). The extent and strength of the cyclonic gyre is variable, as it can extend beyond the limits of the Gulf of Honduras and reach $85^{\circ} \mathrm{W}$ on the Honduras coast, recirculating water from Honduras to Belize (Chérubin et al. 2007). Daily outputs of the first 5 layers (i.e. from 0 to $100 \mathrm{~m}$ ) of the ROMS simulations were inputs to the offline larval tracking model. Vertical velocities, $W$, were not incorporated in the particle motion.

Particle-tracking module. Individual particle movements are tracked offline with LSM, assuming that the evolution of particle velocity and position in nonhomogeneous, non-stationary turbulence can be represented as a Markovian process (Griffa 1996). We used different LSM configurations to test the effect of spatial and temporal averaging of Lagrangian parameters (i.e. decorrelation time scale $T_{\mathrm{L}}$ i horizontal variance of the velocity $\sigma^{2}=\left\langle u^{\prime 2}\right\rangle$, where $<>$ is a spatial or temporal average; spin parameter $\Omega$ allowing for the vorticity of the eddy field; Veneziani et al. 2005a,b) on larval dispersal estimates. Our baseline model was Markovian for $(x, u)$, the particle position and velocity field, respectively, and its governing equations were:

$$
\begin{gathered}
\mathrm{d} x_{i}=\left[\left\langle u_{i}(x, y, z)>+u_{i}{ }^{\prime}\right] \mathrm{d} t+\mathrm{d} u_{i}{ }^{\prime} \mathrm{d} t\right. \\
\mathrm{d} u_{i}{ }^{\prime}=\left[-u_{i}{ }^{\prime} / T_{L}+a\left(x, y, z, u_{i, j}{ }^{\prime}\right)\right] \mathrm{d} t+b_{i}(x, y, z) \mathrm{d} W(t)
\end{gathered}
$$

where $i$ is 1 or 2 ( $x$ and $y$ directions), $d x$ is the particle displacement and $\mathrm{d} u^{\prime}$ is the velocity increment of the turbulent velocity at each time step. The first and second terms on the right-hand side of Eq. (1) are the mean velocity and the turbulent velocity, respectively, $\mathrm{d} t$ is the time step, and $(x, y, z)$ are the coordinates. The first term on the right-hand side of Eq. (2) represents a fading memory for velocity fluctuations; the second term, $a$, the drift correction term, is zero when turbulence is stationary and homogeneous (Berloff \& McWilliams 2002). The third term represents random forcing, where $\mathrm{d} W$ is a random increment from a Wiener process (i.e. continuous-time Gaussian stochastic process) with zero mean and variance $\mathrm{d} t$ and $b$ is the amplitude of the random increment (Berloff \& McWilliams 2002). Applied to larval transport, $b$ could also describe larval swimming (e.g. oriented motion; see Codling et al. 2004). Some measure of the rotation of trajectories is necessary to account for subdiffusive processes, driven, for instance, by submesoscale coherent vortices. Reynolds (2002a) coined the measure of rotation the 'spin' parameter $(\Omega)$ and introduced it in the LSM. Thus, the drift correction term $a$ is associated with the spin parameter $\Omega$ (Borgas et al. 1997, Reynolds 2002a, Veneziani et al. 2004), and the amplitude of the random forcing is a function of the horizontal variance of the velocity field. The velocity increment equations were: 


$$
\begin{aligned}
& \mathrm{d} u^{\prime}=-\frac{u^{\prime}}{T_{L u}} \mathrm{~d} t-\Omega v^{\prime} \mathrm{d} t+\left(\frac{2 \sigma_{u}^{2}}{T_{L u}}\right)^{1 / 2} \mathrm{~d} W_{u}(t) \\
& d v^{\prime}=-\frac{v^{\prime}}{T_{L V}} \mathrm{~d} t+\Omega u^{\prime} \mathrm{d} t+\left(\frac{2 \sigma_{V}^{2}}{T_{L V}}\right)^{1 / 2} \mathrm{~d} W_{v}(t)
\end{aligned}
$$

Here, the eddy diffusivity is $K=\left(2 \sigma^{2} / T_{\mathrm{L}}\right)^{1 / 2}$, which is $b$ in Eq. (2). The Lagrangian parameters of the biophysical model (i.e. $T_{\mathrm{L}}, \sigma$, and $\Omega$ ) were directly estimated from the Eulerian fields of the ocean model using Middleton's relationships between the Eulerian and Lagrangian decorrelation spatial and temporal scales (Middleton 1985, Lumpkin et al. 2002) such that:

$$
T_{\mathrm{L}} / T_{\mathrm{E}}=q\left[q^{2}+\left(u^{\prime} / c_{*}\right)^{2}\right]^{-1 / 2}
$$

where $\mathrm{q}=\sqrt{\pi / 8}$. The ratio $C_{*} \equiv L_{\mathrm{E}} / T_{\mathrm{E}}$ is the evolution speed of the eddy field constructed from its Eulerian time $\left(T_{\mathrm{E}}\right)$ and length scales $\left(L_{\mathrm{E}}\right)$. The parameters $u^{\prime}$ and $v^{\prime}$ (in the $x$ and $y$ directions) are the root mean square eddy speed calculated from the Lagrangian statistics using float trajectories.

The spin obtained from the direct calculation of the Eulerian mean rate of rotation $\langle d s\rangle$ :

$$
\Omega=\frac{\langle d s\rangle}{2 \mathrm{~d} t\left(u^{\prime 2}+v^{\prime 2}\right)}
$$

This model was constrained by the well-mixed condition criterion (Thomson 1987), which implies that a passive tracer uniformly mixed over the full domain remains uniformly mixed at all times. In this classical form of the LSM model, the Lagrangian parameters (i.e. $T_{\mathrm{L}}$ $\sigma^{2}$, and $\Omega$ ) are then spatially uniform or constant in time. However, the definition of $\Omega$ accounts for the spatial variation of the variance of the velocity field; thus, TL and $\sigma^{2}$ can be spatially variable if subregions made of homogeneous statistical properties are defined (Lumpkin et al. 2002, Veneziani et al. 2004). Moreover, to improve the capabilities of the LSM in simulating a broader range of intermediate-time, non-diffusive, single-particle timedispersion behaviors involving a variety of time scales and length scales, we followed Berloff \& McWilliams' (2003) method to randomize our LSM, by defining a distribution of each variable of the triplet $\left(T_{\mathrm{L}} \sigma^{2}\right.$, and $\Omega$ ) in individual bins.

Biological module-stochastic ontogenetic vertical migration and mortality. The vertical distribution of larvae is time dependent. We built species-specific matrices of larval probability vertical distribution with time, based on field observations combined with otolith analyses to age the larvae (Paris-Limouzy 2001). Here, we modelled 2 types of migration patterns, the 'deep' and 'shallow' ontogenetic vertical migrations (OVMs) analogous to those of damselfish (Pomacentridae; Fig. 4) and grouper (Serranidae; see Fig. 3 in Cowen 2002) larval behavior, respectively. The code reads the probabilities for the vertical distribution of a given species $P_{\text {spec }}(l, z)$ and converts the probabilities into a random number $0<R_{\mathrm{N}}<1$ by solving the integral equation for $z_{i}$ :

$$
R_{\mathrm{N}}\left(1, z_{i}\right)=\int_{-\infty}^{z_{i}} P_{\text {spec }}(1, z) \mathrm{d} z
$$

where $l$ is the duration of the stage-specific vertical distribution (e.g. $3 \mathrm{~d}_{i}$ see Fig. 4 ) and $z_{i}$ is the depth where the larvae are found. The frequencies of the resulting depth values $z_{i}$ reflect the characteristics of the probability distribution.

The mortality rate can be supplied either as a constant value or as a 4-dimensional Eulerian field $(x, y, z$, time), whereby its value close to an individual larval location is used to calculate the probability of mortality. Here, we used a constant mortality rate assigned stochastically among particles (i.e. patchy mortality). Dead larvae were flagged and removed from further calculations.

Seascape module. The seascape module serves to quantify the ecological interactions (e.g. particle behavior, exchange of particles) at the boundary of the pelagic and coral reef ecosystems. Coupled to the particle-tracking module, it traces the source location and provides habitat information to the particle (e.g. presence of suitable nursery areas) at each time step. The habitat (i.e. spawning and settlement areas) is derived from remote sensing of reef-building corals (Burke \& Maidens 2004) and is buffered with a sensory zone representing the ability of reef fish larvae to sense and swim towards settlement habitat (Fisher et al. 2000,

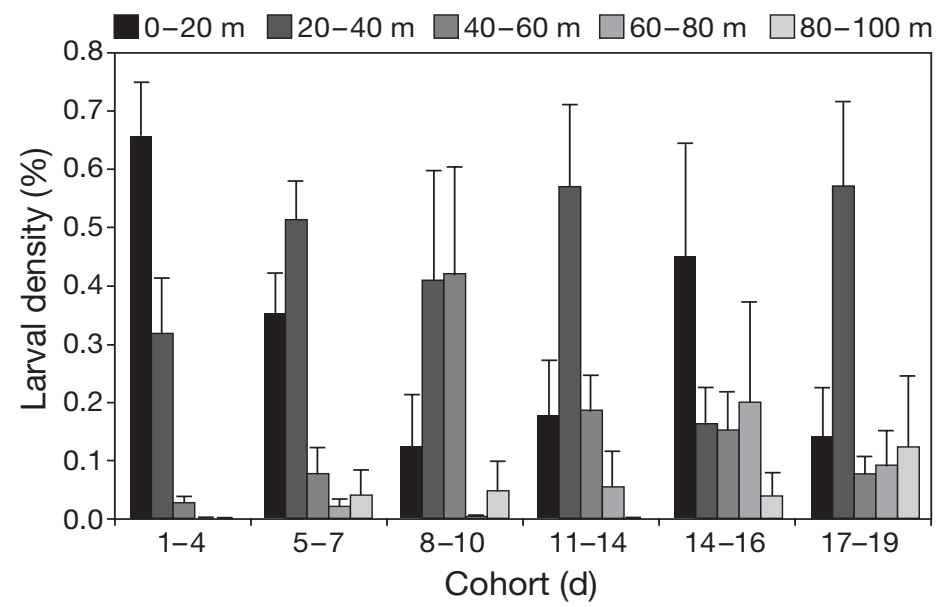

Fig. 4. Stegastes partitus. Distribution of depth-specific density frequency of $3 \mathrm{~d}$ cohorts (based on otolith daily increments) for larval damselfish captured off the west coast of Barbados during May 1996 and May 1997 with a $1 \mathrm{~m}^{2}$ Multiple Opening and Closing Net and Environmental Sampling System (MOCNESS). The bicolor damselfish mean larval duration is $29 \mathrm{~d}$, but larvae older than $19 \mathrm{~d}$ were too scarce for analysis. Error bars represent the variance (SE) in the density of larvae in each 5 nets of the 448 MOCNESS tows ( $\mathrm{n}=2240$ samples) by Paris-Limouzy (2001) 
Fisher \& Bellwood 2002, Gerlach et al. 2007). The seascape habitat is further parted into sections of similar reef areas (e.g. nodes) that serve to build the connectivity matrix. We used a $9 \mathrm{~km}$ sensory zone and ca. $50 \mathrm{~km}$ reef sections. The model domain is the southern meso-American region (15 to $21^{\circ} \mathrm{N}, 84$ to $89^{\circ} \mathrm{W}$; Fig. 3 ).

Model output-connectivity matrix. The likelihood of larval exchange from one population to another was represented in a transition probability matrix, where columns are source reefs (node $i$ ) and rows are destination reefs (or node $j$ ). The content of a given matrix element describes the probability of an individual larva making the transition from its source population and successfully reaching the settlement stage in the destination population. Elements along the diagonal of the matrix (where source = sink) represent self-recruitment within a population. Connections between populations may be represented by several types of matrices: (1) the distance matrix $d_{i j}$ represents the distances between reefs $i$ and $j_{i}(2)$ the transition probability matrix $P_{i j}$ represents the probability that an individual larva in node $i$ at time $t$ will disperse to node $j$ at time $t+k$, where $k$ is the pelagic larval duration; (3) the adjacency matrix (or edge) $A=a_{i j}$ is a binary matrix in which each element is defined as $a_{i j}=1$, if nodes $i$ and $j$ are connected, otherwise $a_{i j}=0$. This matrix is mostly used to analyze connectivity networks (Urban \& Keitt 2001). The expected flux $F$ from node $i$ to node $j$ is:

$$
F_{i j}=S_{i} / S_{\text {tot }} \times P_{i j}
$$

where $S_{i}$ is the size of the population in node $i$ and $S_{\text {tot }}$ equals $\sum S_{i}$. We set $S_{i}$ to be constant, corresponding with uniform particle release at all locations.

\section{NUMERICAL EXPERIMENTS AND ANALYSES}

Two types of numerical experiments were carried out: (1) an experiment that compared several types of offline configurations with online calculations and (2) an experiment with the 'best' offline model, but with different biological attributes (e.g. behavior and mortality).

The ROMS was first used to diagnose the oceanographic field by calculating the Lagrangian statistics to be used in the offline LSM configurations. For this purpose, 2 nested simulations were used, the parent and the child grids, their respective resolution and inte- gration time steps being $6 \mathrm{~km} / 720 \mathrm{~s}$ and $2 \mathrm{~km} / 240 \mathrm{~s}$ (Fig. 3). The high-resolution simulation was used to account for the unresolved subgrid-scale processes of the coarser grid simulation where they overlap. The Lagrangian parameters obtained constrain the various LSM configurations used in this sensitivity study as described below (Fig. 5). The time step used for the LSM was $1 \mathrm{~d}$, which is standard to ocean circulation model archives. Such a time step prevents the resolution of some eddies, as the mean speed is enough to prevent particles from being trapped by coherent structures. By introducing the spin parameter, the particle-trapping effect of eddies is better resolved. At smaller time steps, more and smaller eddies are resolved, which might be sufficient to simulate particle trapping by eddies without the spin parameter, but this issue was not addressed in the present study, since online tracking was used most of the time for $<1 \mathrm{~d}$ time steps. Online ROMS surface drift (or passive scenario) simulations were used as a 'null' model (Model 0) to estimate how the various offline LSM configurations perform. A random turbulent velocity term was computed to parameterize unresolved subgrid scales, which is the same as in the offline model, where $b$ is calculated as in Eqs. (3) and (4). For the coarse grid (6 km), the eddy diffusivity is $K=7.4 \mathrm{~cm}^{2} \mathrm{~s}^{-1}$, and in the high-resolution grid $(2 \mathrm{~km}), K=5.1 \mathrm{~cm}^{2} \mathrm{~s}^{-1}$.

\section{Numerical Experiment 1}

To estimate the relative effect of the spatial average of the Lagrangian parameters, several averaging methods were used: we defined bins of $20 \times 20$ grid points, where the model statistics are considered homogeneous and the Lagrangian parameters are estimated to fulfill the well-mixed condition. Each bin was associated with a distribution of $T_{\mathrm{L}}, \sigma$, and $\Omega$ made of the values calculated at each grid point of the bin. The distribution was assumed to be normal in order to fit with the LSM definition. The distribution of $\Omega$ was time dependent. In the first configuration (Model 1), 1 triplet of $\left(T_{\mathrm{L}}, \sigma\right.$, and $\Omega$ ) was randomly selected at each time step of the LSM and for each particle; in the second configuration (Model 2), $T_{\mathrm{L}}$ and $\sigma$ were constant per bin, while $\Omega$ was a time-dependent distribution; in the third configuration (Model 3), $T_{\mathrm{L}}$ and $\sigma$ were constant

Fig. 5. Spatial anisotropy of (A) the flow field variance and (B) the Lagrangian decorrelation time scale $T_{\mathrm{L}}$ from the Regional Ocean Modeling System (ROMS) at $6 \mathrm{~km}$ (parent grid) and $2 \mathrm{~km}$ (child grid), in the meso-American region from 1 to 30 January of a climatological year. In Panels I and II the parameters are derived from the ROMS Eulerian field for the parent grid, while in Panels III and IV they are derived from $30 \mathrm{~d}$ online float trajectories released along the reef edge; Panels V to VIII are the same as Panels I to IV, but for the child grid; values are averaged over bins of $60 \times 60$ grid-cells in the child grid. Note that the Lagrangian binning captures the dominant values and spatial structure of the Eulerian field along the trajectories 

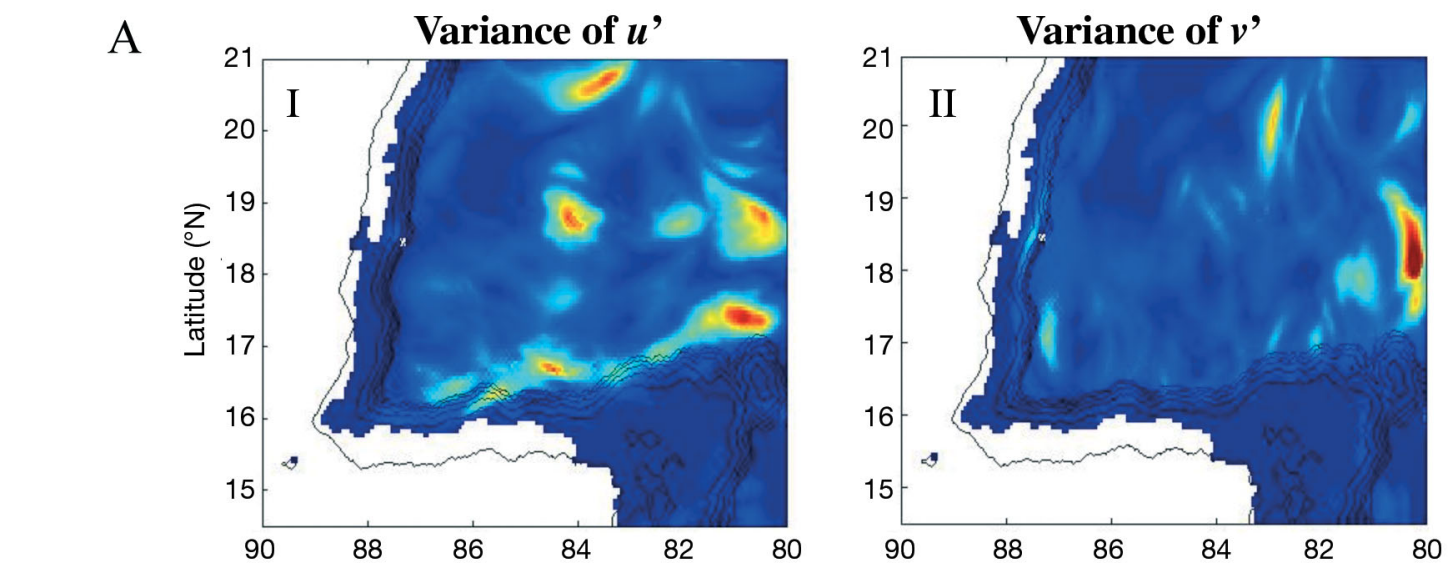

|
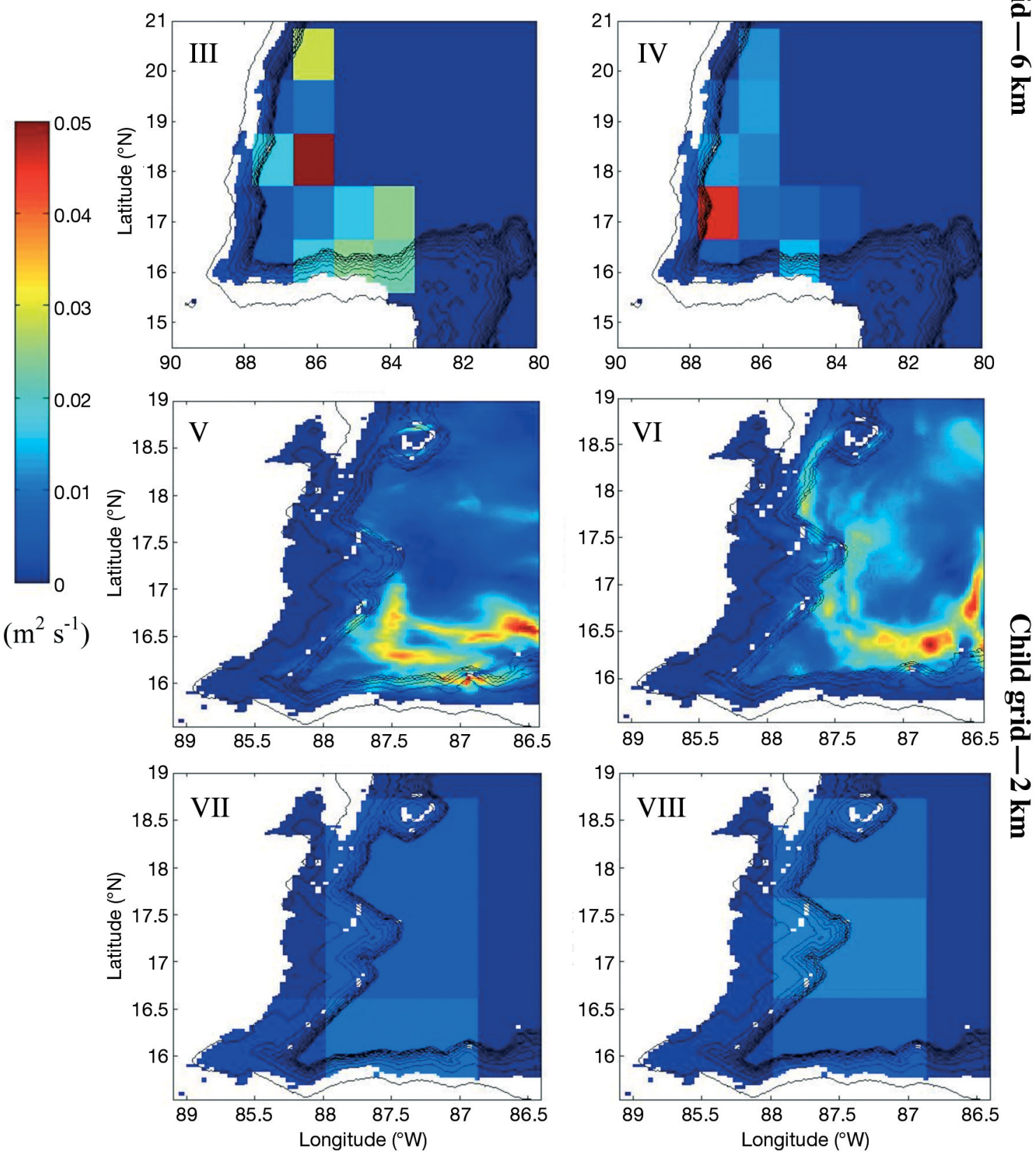

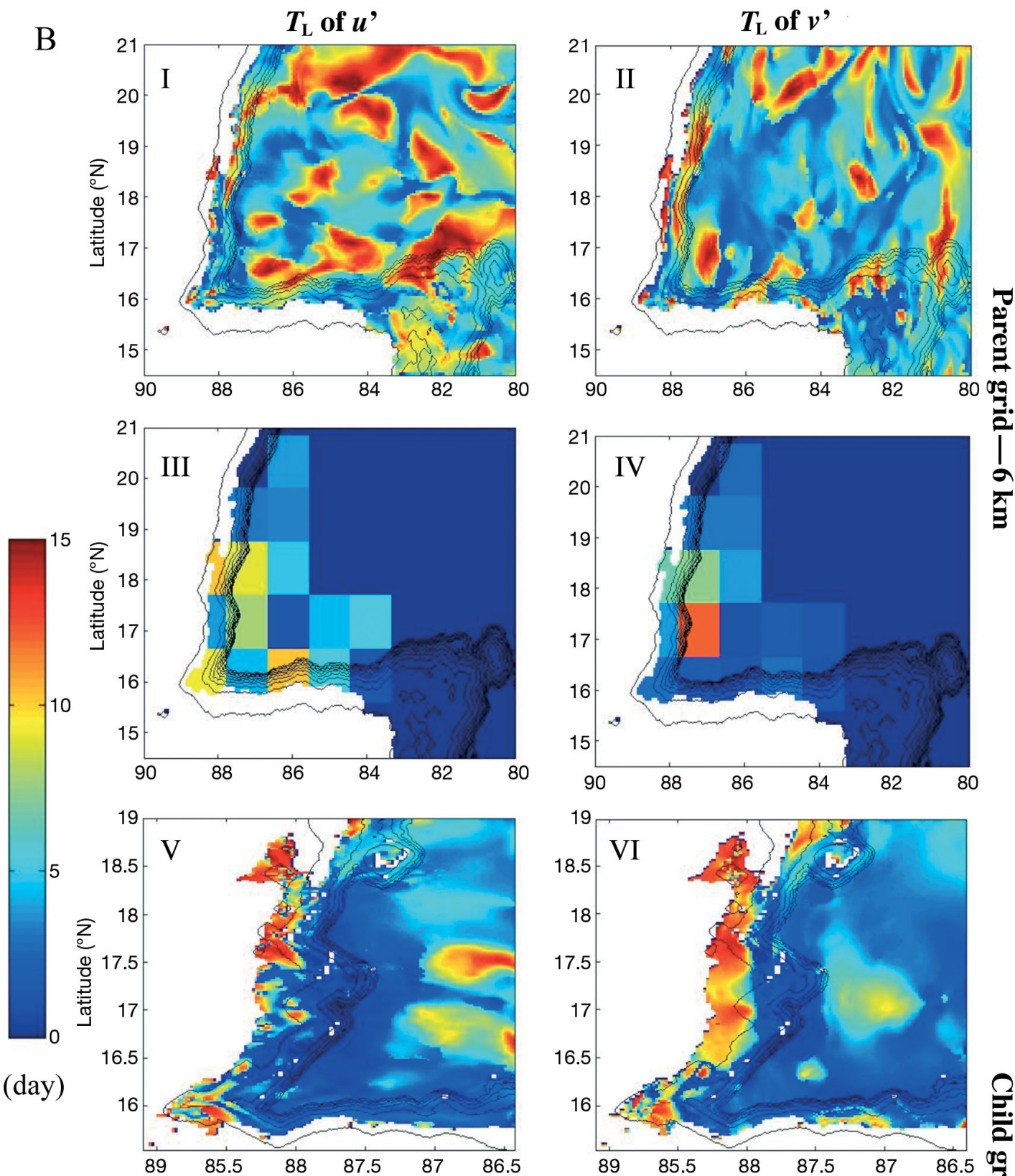

ב⿳亠口冋.
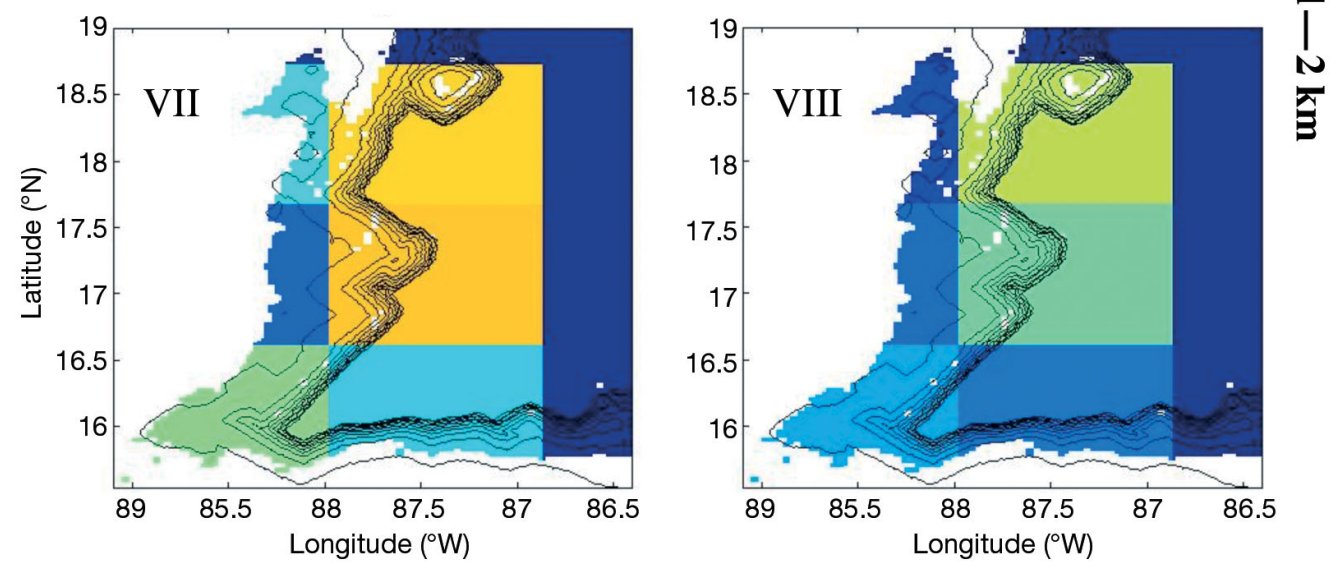
in the entire domain and their values were set by the average of the Lagrangian parameters obtained from the online trajectories and $\Omega=0$ (Fig. 5); in the fourth configuration (Model 4 ), $T_{\mathrm{L}}, \sigma$, and $\Omega$ were obtained from their Eulerian statistics at each grid point. We assumed here that the homogeneous statistics regions are self-defined by the flow field and not arbitrarily by bins as seen in Fig. 5. As the calculation of Lagrangian parameters depends on the estimation of the mean flow, 2 averaging methods are used to calculate the mean flow: the time average at each grid point (Tmn) or time average per $20 \times 20$ grid-cell bin (bin). Also, 2 methods were used to calculate the absolute velocity at the particle location: the velocity at the closest grid point (cgp) or the bilinear interpolation (int). Subgrid-scale nesting was computed in 2 different ways. (1) Combined with Model 1, as each bin of the parent grid contains several bins of the child grid, the statistics of the parent bin and child bins were merged (nest1). (2) Subgrid-scale nesting (nest2), which is combined with Model 4, consists of merging the statistics of a parent grid with those of a child grid; each parent grid point falls in a bin of the child grid $(2 \mathrm{~km})$ where the statistics are calculated as in Model 1. Therefore, the subgrid parameters are obtained from each bin where the distributions of the statistical parameters $T^{1}{ }_{L}, \Omega^{1}$, and $\sigma_{1}$ are also calculated. The turbulent velocity of the subgrid (which contains the turbulent field also parameterized by a random term) was added to the turbulent velocity of each particle in the parent grid. The LSM model equations for the nested model were:

$$
\begin{gathered}
\mathrm{d} u_{0}^{\prime}=-\frac{u^{\prime}}{T_{L}} \mathrm{~d} t-\Omega v^{\prime} \mathrm{d} t \\
\mathrm{~d} u_{1}^{\prime}=-\frac{u^{\prime}}{T_{L}^{1}} \mathrm{~d} t-\Omega^{1} V_{1} \mathrm{~d} t+\sqrt{2 \sigma_{1}^{2} / T_{L}^{1}} \mathrm{~d} W(t) \\
u_{\text {turb }}=u^{\prime}+\mathrm{d} u_{0}^{\prime}+\mathrm{d} u_{1}^{\prime}
\end{gathered}
$$

where $T_{\mathrm{L}}$ and $\Omega$ were calculated at each grid point of the parent grid; $T_{L}^{1}, \Omega^{1}$, and $\sigma_{1}$ are distributions in the child grid $u_{0}^{\prime}$ and $u_{1}^{\prime}$ are the turbulent velocity in parent and child grids, respectively; and $u_{\text {turb }}$ is the new turbulent velocity from subgrid nesting.
Table 1. Lagrangian stochastic model (LSM) configurations. A series of spatial and the Lagrangian statistics used in ine tracking. All parameters $T_{\mathrm{L}}, \sigma$, and $\Omega$ were calculated for $30 \mathrm{~d}$, and 100 par( 48 sites) were released for each run, repeated 8 times. Results are from 2 grid points; pdf: distribution of the ensemble of values calculated from grid-scale nesting by merging the statistics of a parent and child grid; shallow: on-

\begin{tabular}{|c|c|c|c|c|c|c|c|}
\hline $\begin{array}{l}\text { LSM } \\
\text { model type }\end{array}$ & $T_{\mathrm{L}}$ & $\sigma$ & $\Omega$ & $\begin{array}{c}\text { LSM } \\
\text { nesting }\end{array}$ & $\begin{array}{l}\text { Averaging } \\
\text { method }\end{array}$ & OVM & $\begin{array}{c}\text { Mortality } \\
\left(\mathrm{d}^{-1}\right)\end{array}$ \\
\hline 0 & intrinsic & intrinsic & intrinsic & - & - & - & 0 \\
\hline $1 \mathrm{a}$ & pdf & pdf & pdf & - & cgp-bin & - & 0 \\
\hline $1 b$ & pdf & pdf & pdf & nest1 & cgp-bin & - & 0 \\
\hline $1 \mathrm{c}$ & pdf & pdf & 0 & - & cgp-bin & - & 0 \\
\hline $1 d$ & pdf & pdf & pdf & - & cgp-Tmn & - & 0 \\
\hline $1 \mathrm{e}$ & pdf & pdf & pdf & nest1 & cgp-Tmn & - & 0 \\
\hline 1f & pdf & pdf & pdf & - & int-Tmn & - & 0 \\
\hline $1 \mathrm{~g}$ & pdf & pdf & pdf & nest1 & int-Tmn & - & 0 \\
\hline $1 \mathrm{~h}$ & pdf & pdf & pdf & nest1 & cgp-bin & shallow & 0 \\
\hline $1 \mathrm{i}$ & pdf & pdf & pdf & nest1 & cgp-bin & deep & 0 \\
\hline $1 \mathrm{j}$ & pdf & pdf & pdf & nest1 & cgp-bin & deep & 0.05 \\
\hline $1 \mathrm{k}$ & pdf & pdf & pdf & nest1 & cgp-bin & - & 0.05 \\
\hline 11 & pdf & pdf & pdf & nest1 & cgp-bin & - & 0.1 \\
\hline $1 \mathrm{~m}$ & pdf & pdf & 0 & nest1 & cgp-bin & deep & 0 \\
\hline $2 a$ & $\mathrm{C}_{\mathrm{B}}$ & $\mathrm{C}_{\mathrm{B}}$ & pdf & - & cgp-bin & - & 0 \\
\hline $2 b$ & $\mathrm{C}_{\mathrm{B}}$ & $\mathrm{C}_{\mathrm{B}}$ & pdf & - & cgp-Tmn & - & 0 \\
\hline $2 \mathrm{c}$ & $\mathrm{C}_{\mathrm{B}}$ & $\mathrm{C}_{\mathrm{B}}$ & pdf & - & int-Tmn & - & 0 \\
\hline $2 d$ & $\mathrm{C}_{\mathrm{B}}$ & $\mathrm{C}_{\mathrm{B}}$ & pdf & - & int2-Tmn & - & 0 \\
\hline $3 a$ & $\mathrm{C}_{\mathrm{D}}$ & $C_{D}$ & pdf & - & cgp & - & 0 \\
\hline $3 b$ & $\mathrm{C}_{\mathrm{D}}$ & $\mathrm{C}_{\mathrm{D}}$ & 0 & - & $c g p$ & - & 0 \\
\hline $3 \mathrm{c}$ & $C_{D}$ & pdf & 0 & - & cgp-bin & - & 0 \\
\hline $3 d$ & pdf & $\mathrm{C}_{\mathrm{D}}$ & 0 & - & cgp-bin & - & 0 \\
\hline $4 a$ & cgp & cgp & 0 & - & cgp-Tmn & - & 0 \\
\hline $4 \mathrm{~b}$ & cgp & cgp & 0 & nest2 & cgp-Tmn & - & 0 \\
\hline
\end{tabular}
togenetic vertical migration (OVM) of damselfish type (Paris-Limouzy 2001); deep:

OVM of grouper type (Fig. 3 in Cowen 2002)

These offline LSM configurations are listed in Table 1. The 'best' configuration was evaluated based on larval (particle) connectivity patterns. Two-dimensional correlation coefficients $(r)$ were computed between the probability transition matrices $A_{i j}$ (i.e. the probability that an individual larva from $i$ will disperse to $j$ ) generated by the offline models (Models 1 to 4 ) and the online model (Model 0, reference matrix $O_{i j}$ ):

$$
r=\frac{\sum_{i} \sum_{j}\left[\left(A_{i j}-\hat{A}_{i j}\right) \times\left(O_{i j}-\hat{O}_{i j}\right)\right]}{\sqrt{\left[\sum_{i} \sum_{j}\left(A_{i j}-\hat{A}_{i j}\right)^{2} \times \sum_{i} \sum_{j}\left(O_{i j}-\hat{O}_{i j}\right)^{2}\right]}}
$$

The higher the correlations with the offline reference matrix, the better the fit (Fig. 6). The configuration that perform the best was selected for the second numerical experiment on biological attributes. 


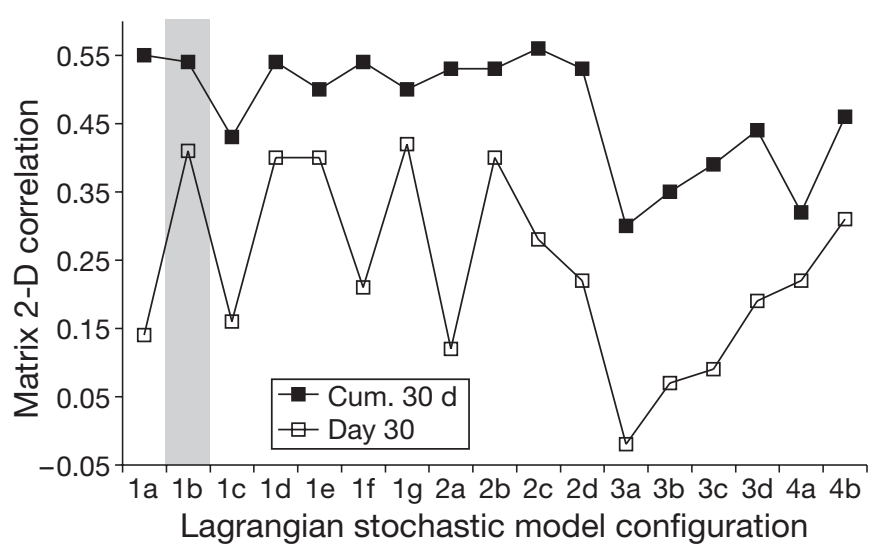

Fig. 6. Sensitivity analysis on the performance of the offline Lagrangian stochastic model (LSM) configurations: patterns of connectivity simulated by the offline models are weighted against the online model's (Model 0). The higher the correlation ( $y$-axis), the better the fit of the offline model for particle position at the end of $30 \mathrm{~d}$ passive dispersal (Day 30) and for cumulative arrivals during the $30 \mathrm{~d}$ integration (Cum. $30 \mathrm{~d}$ ). Parameterization of the offline LSM configurations is shown in Table 1; shaded area indicates the best offline performance

\section{Numerical Experiment 2}

The calibrated offline model served to control the physical and biological parameters invoked in the particle-tracking scheme (i.e. Models $1 \mathrm{~h}-1 \mathrm{~m}$ in Table 1). The relative effect of the biophysical factors and their interactions on connectivity patterns was classified by computing the 2-dimensional correlation coefficients between the transition probability matrices generated with a series of biological (i.e. behavior and mortality) and physical (i.e. eddy field) attributes and a $30 \mathrm{~d}$ passive surface drift scenario. The lower the correlation with the offline reference matrix, the greater the behavioral effect on dispersal. We quantified how much single and combined factors depart from the simplified assumption (i.e. passive dispersal and no mortality).

These numerical experiments were based on $30 \mathrm{~d}$ runs, to reflect a mean pelagic larval duration common among coral reef fish (Lindeman et al. 2005, Paris et al. 2005a). Due to the stochastic nature of the models, we obtained a slightly different matrix for each reiteration of the same model configuration. Therefore, each LSM model was run 8 to 10 times.

\section{SIMULATION RESULTS}

\section{Choice of the Lagrangian stochastic model}

For the configurations of the offline LSM with constant velocity variance and Lagrangian time scale, we obtained clustered trajectories and a sparse matrix that were poorly correlated with the online output (Figs. 6 \& 7). When $T_{\mathrm{L}}$ and $\sigma$ were constant over the entire domain, the spread and length of the trajectories depended on the arbitrary choice of these values; adding a spin parameter did not improve the fit. Introducing spatially and temporally explicit parameters help to reproduce both trajectories and patterns of larval exchange (Fig. 7). The various configurations of spatial and temporal averaging of the parameters used in the offline LSM model had little effect on cumulative arrivals over the $30 \mathrm{~d}$ simulation period, although instantaneous settlement on Day 30 differed (Fig. 6). The 'best' model configuration emerged from the nested configurations with spin, as they tended to better resolve subgrid-scale processes. The nested-bin model with spin (i.e. Model Type 1b; Table 1) was thus selected for the biophysical models in Numerical Experiment 2.

\section{Influence of physical and biological parameters on dispersal distances}

The reef location of successful simulated larvae was closer to the natal reef when behavior was invoked (Fig. 8A), even though total displacements were well conserved in all the models (Fig. 8B). In addition, levels of both total and self-recruitment were increased with vertical migration and eddy field, while they were depressed with mortality by an order of magnitude, albeit the mortality rate was in the low range of observed values (i.e. $0.03<m<0.52$; Houde 1989). Interestingly, the tail of the dispersal kernel was greatly reduced with mortality. Finally, dispersal distances were always increased, and the level of selfrecruitment decreased in models where $T_{\mathrm{L}}$, and/or $\sigma$ were kept constant over the entire domain (data not shown, Model 3).

\section{Relative influence of biological and physical parame- ters on connectivity}

Biological parameters (i.e. ontogenetic vertical migration and mortality) and physical parameters (i.e. spin or eddy field) changed the connectivity pattern from that of simple drift (Fig. 9). Pairwise comparison of the means of matrix correlations for each model configuration indicated how much each factor departs from the others. For example, 'deep' ontogenetic vertical migration combined with low mortality (Model 1j) differed significantly from passive transport with no mortality (Model 1b) or high mortality (11), but not from the other scenarios. The model with higher mortality (11) showed the largest divergence from passive drift and no mortality (1b). When compared to passive drift (1b), onto- 

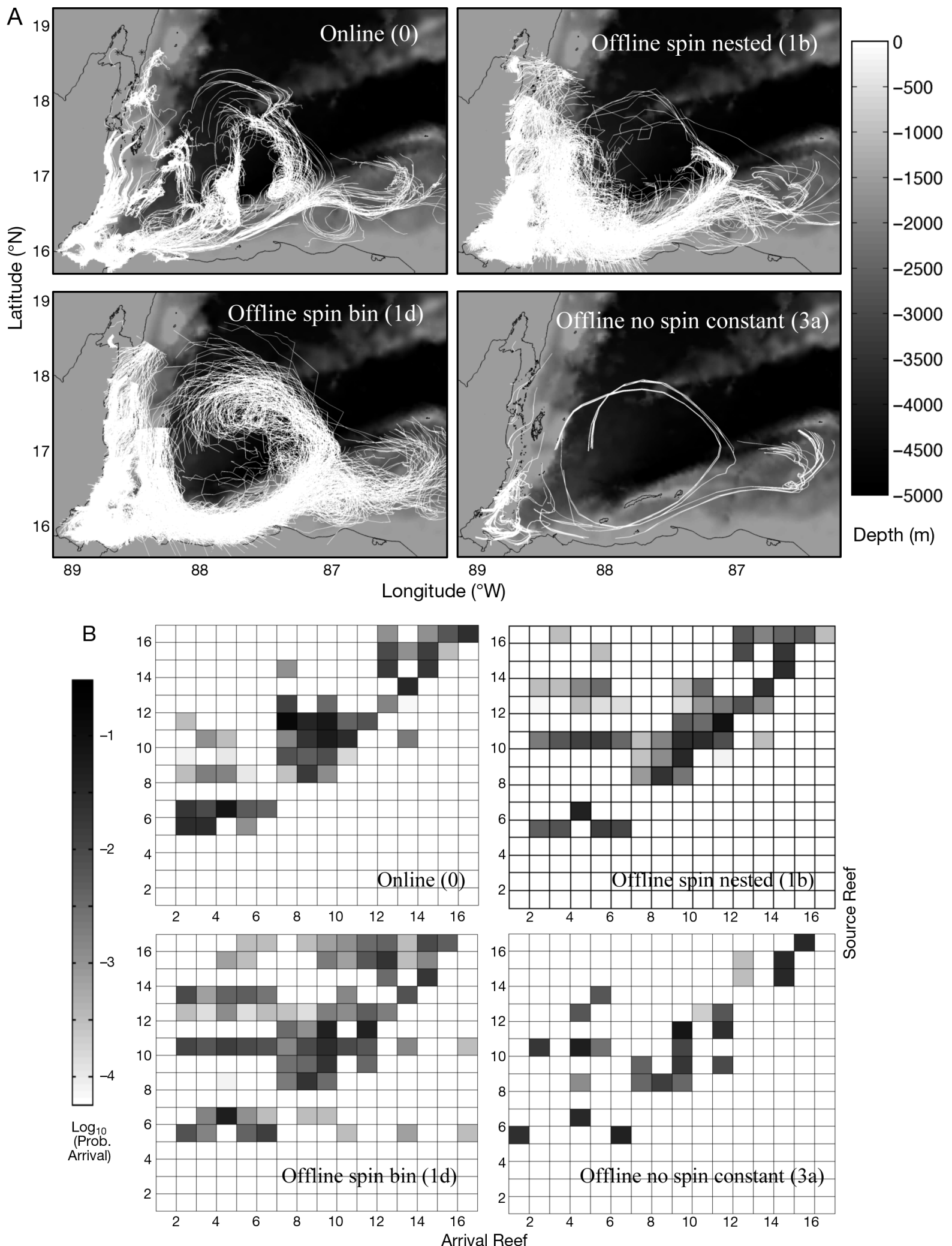

Fig. 7. Online-offline comparison of (A) trajectories and (B) connectivity matrices for $30 \mathrm{~d}$ passive dispersal of 100 particles released from 48 reef locations in the meso-American region in January of a climatological year, by ROMS. Parameterization of the Lagrangian stochastic model configurations is shown in Table 1. Note that there seem to be fewer trajectories in the online model (0), since particles are absorbed at the land mask, whereas they are reflected in the offline model 


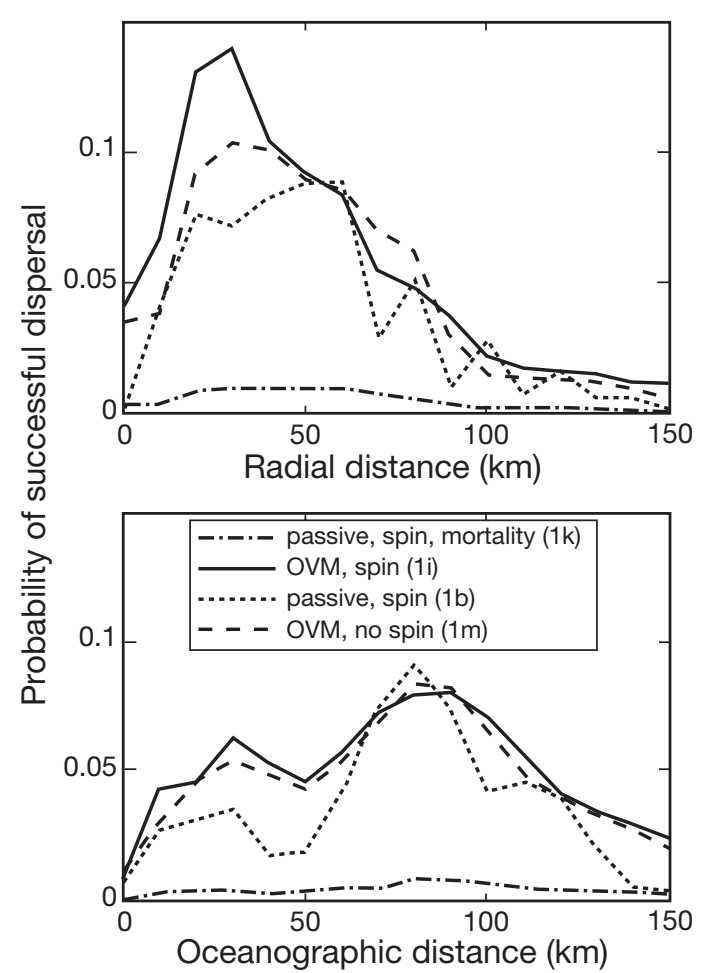

Fig. 8. Probability of successful dispersal distance (or dispersal kernel) measured as (A) radial distance from the source population and (B) oceanographic distance (or distance that the particle traveled along its trajectory). The mean dispersal kernels for a $30 \mathrm{~d}$ pelagic larval duration are derived from a series of offline model runs (8 iterations of 100 particles released at 48 locations) controlling the behavior of simulated larvae (i.e. ontogenetic vertical migration, OVM), as well as from eddy field parameterization (i.e. spin). While modes of oceanographic distances (i.e. $80 \mathrm{~km}$ ) are similar for all the models, modes of radial distances are longer for those configured without resolving small eddies, i.e. modal distance of $40 \mathrm{~km}$ for OVM with no spin (Model $1 \mathrm{~m}$ ) vs. $20 \mathrm{~km}$ for OVM with spin (Model 1i; $\mathrm{p}<0.05$ ). Mortality depresses the dispersal kernels, while self-recruitment increases with larval behavior

genetic vertical migration changed significantly the patterns of larval exchange in the 'deep' (1i), but not in the 'shallow' (1h), scenario (Fig. 9). Yet, recruitment levels were augmented in 'shallow' vertical movement (Fig. 8A). A similar trend emerged from monthly simulations over the entire Caribbean (Fig. 10), where, in most cases, recruitment increased with the implementation of 'shallow' vertical migration during larval development (Fig. 11), e.g. direction of dispersal (e.g. St Croix), connectivity pattern (e.g. Montego Bay, Jamaica), and self-recruitment may change (e.g. Florida Keys and Los Roques, Venezuela). Increased survival with such behavior is mostly evident for selfrecruitment even for short larval durations. Vertical migration became a significant factor in terms of raising the levels of subsidies when pelagic larval duration was increased (Fig. 10).

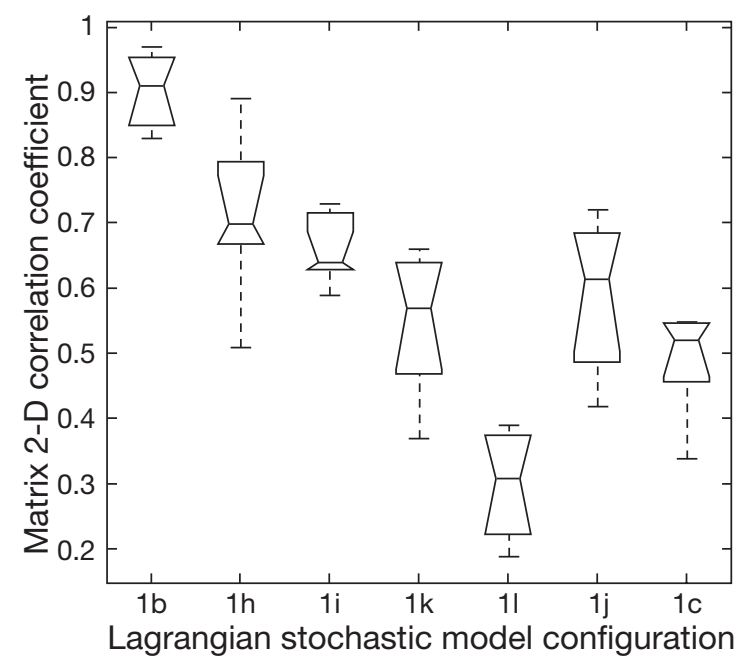

Fig. 9. Relative influence of biological and physical factors on population connectivity estimates: similarity of transition probability matrices derived from varying biophysical model configurations (i.e. Model 1, see Table 1) is scored against passive transport (or surface drift), spin, and no larval mortality. Each model configuration was run 8 times and SE (dotted bars), median (narrow part of box), upper and lower quartiles (skew, upper and lower part of box) of the correlation coefficients are depicted by the box plot. One-way analysis of variance indicates that the means of the group are unequal $\left(p=3.36 \times 10^{-11}\right)$

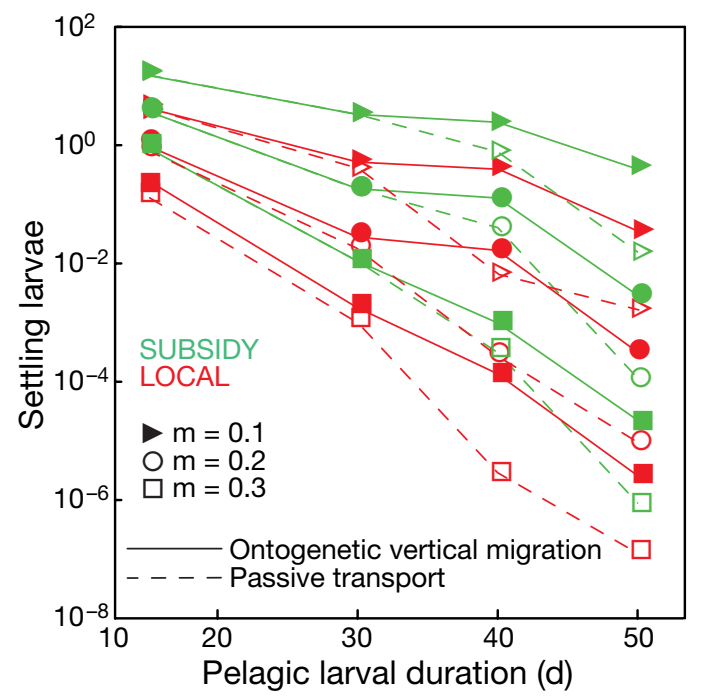

Fig. 10. Influence of life-history traits on recruitment success for subsidy (non-locally produced, $\Sigma P_{i j}$, when $i \neq j, P_{i j}$ being the probability that a larva in node $i$ will disperse to node $j$ ) and self-recruits (locally produced, $\Sigma P_{i j}$, when $i=j$ ) compared to the passive transport of inert particles. Life-history traits are: pelagic larval duration, daily larval mortality rate $(\mathrm{m})$, and ontogenetic vertical migration (solid lines) using the 'shallow' scheme observed in damselfish Stegastes partitus. The sensitivity analysis is based on 24 runs, each consisting of 1560000 trajectories $(260$ locations $\times 12$ release times $\times 500$ particles) throughout the entire Caribbean basin (see Fig. 1 in Cowen et al. 2006). Results from passive transport (dotted lines) are plotted for comparison 

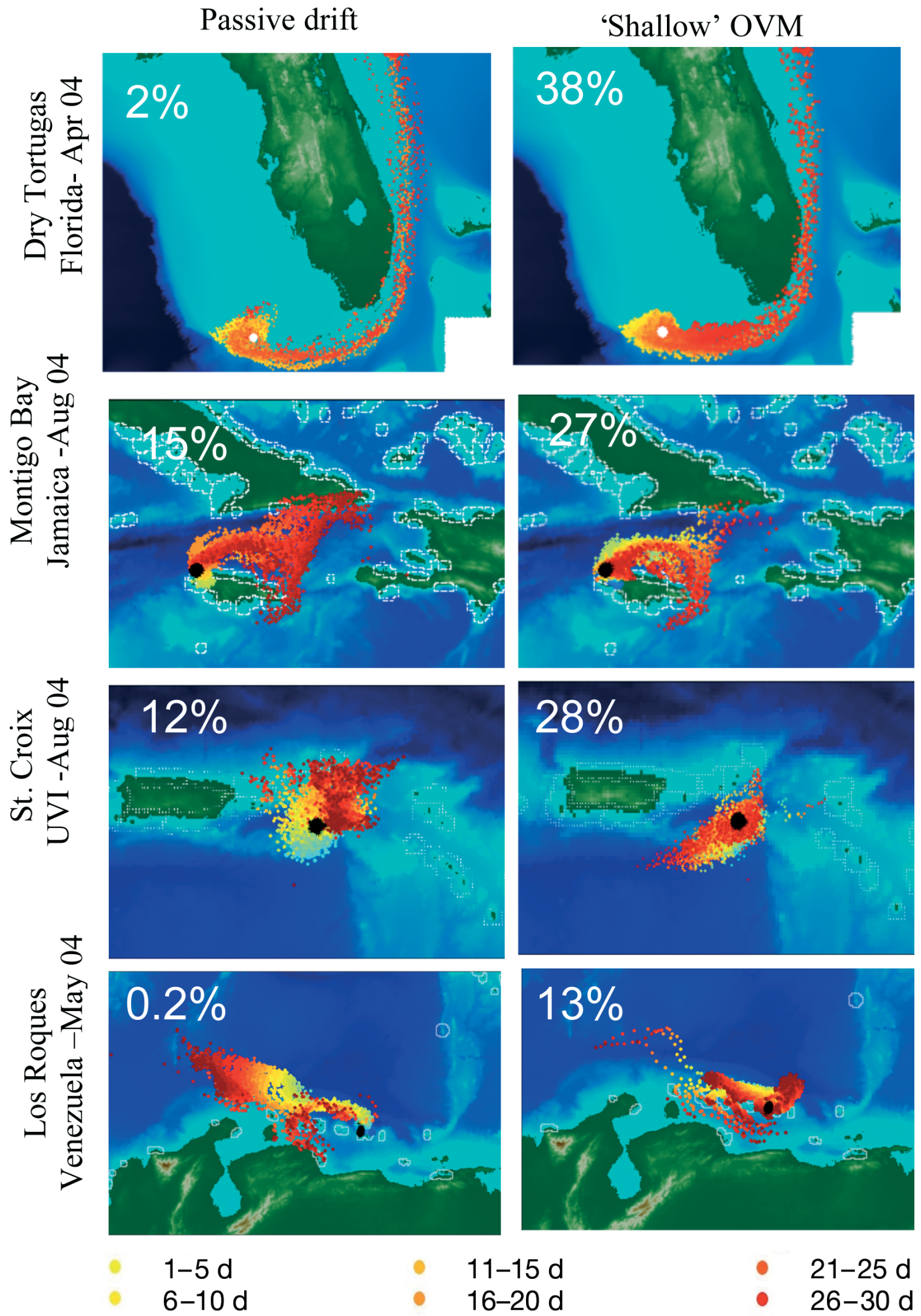

$\begin{array}{ll} & 21-25 \mathrm{~d} \\ & 26-30 \mathrm{~d}\end{array}$

Fig. 11. Influence of larval behavior on dispersal: $30 \mathrm{~d}$ passive dispersal snapshots from various locations in the Caribbean are compared to dispersal with 'shallow' ontogenetic vertical migration (OVM) of the bicolor damselfish Stegastes partitus. Percent of simulated larvae arriving onto any reef is indicated 


\section{DISCUSSION}

Currently, offline LSMs often assume homogeneity of the Lagrangian parameters, and turbulent motion or the eddy diffusivity is typically scaled by the horizontal diffusion of the appropriate grid size according to Okubo (1971). However, it may not represent the real processes of dispersion and mixing. True eddy perturbations occurring at the subgrid scale can vary both spatially and temporally (e.g. cross-shore, with different oceanographic regimes). We provided a simple and effective technique to calculate the Lagrangian parameters $T_{\mathrm{L}}, \sigma$, and $\Omega$ that control the dispersion from the Eulerian field intrinsic to the online hydrodynamic model. With this approach, any other source of Lagrangian statistics, such as real float measurements, can be integrated into the LSM equation. A major improvement of LSMs is the addition of the spin parameter $\Omega$, which accounts for the properties of the eddy field. However, accounting for this eddy-resolving parameter is incumbent upon the time step of the OGCM outputs and the size of the eddies present in the study area. For smaller time steps, this parameter might become obsolete. This effect was not addressed here since most of the OCGM output time steps are $1 \mathrm{~d}$ or more. The spin parameter contributes to looping trajectories, and the LSM is then capable of simulating correctly both suband super-diffusive behaviors in the mean spreading of particles (Reynolds 2002b, Veneziani et al. 2004). As a result, the LSM reproduces the effects of rotating coherent structures such as vortices and mesoscale eddies, which are prominent at the shelf break and around oceanic islands and atolls.

Both ontogenetic vertical migration and mortality rates have a significant effect on the patterns of connectivity. When the mortality rate increases, the transition matrix becomes sparser. Hence, connectivity patterns are more stochastic at each run repetition. Therefore, an ensemble of runs is necessary to produce a 'saturation' curve on the number of possible patterns generated. While increasing mortality rates may not necessarily change the patterns of connectivity, spatially explicit mortality rates may. This option still needs to be explored. Such an exercise would not be of great use without empirical knowledge of the clines in mortality for reef fish larvae during ontogeny. More prey-predator field surveys are urgently needed in tropical ecosystems to understand the behavior of coral reef fish larvae in relation to predation avoidance and feeding (see Fiksen et al. 2007, this TS). Kobayashi (2006) recently suggested that long-distance transport exceeds local retention at longer pelagic durations. However, larvae with longer pelagic durations are subject to daily natural mortality rates for a longer period; which changes recruitment levels by 1 or more orders of magnitude. Mortality due to physical loss may vary with the seascape, combined with larval duration, while total natural mortality always increases with pelagic time.

Determining the levels of recruits is necessary to assess the nature of the connectivity (Cowen et al. 2006). If the number of settling individuals is sufficient to sustain a given local population, the connectivity is 'demographic'. Mortality is the single most imperative factor when assessing levels of demographic exchanges (Cowen et al. 2000, 2006). Indeed, levels of larval exchange are extremely sensitive to small changes in mortality rates (Fig. 9).

Sensory capabilities could also contribute to changing connectivity patterns. Here, we used a constant sensory zone, which was scaled by the parent grid of the ocean circulation model. Yet, sensitivity analysis on the sensory halo by Paris et al. (2005b) suggested that levels rather than spatial patterns of recruitment were affected in the western Caribbean. Baums et al. (2006) found that patterns of connectivity are significantly affected by the onset of active movement in the eastern Caribbean, but not in the western Caribbean. They attributed this to the very distinct geomorphology of these 2 regions: one much more patchy and more naturally fragmented than the other. In the meso-American region examined in this study, with continuous coastlines and offshore atolls, levels of recruitment changed with the duration of the pre-competent period, while patterns of connectivity were not significantly different. Thus, configuration of the recruitment habitat seems to dictate the relative influence of larval traits on connectivity patterns and spatial autocorrelation should be explored to quantify the seascape effect. More work is also needed to assess the spatial scale of the sensory envelope.

Habitat patchiness and natural fragmentation have been identified as major sources of variation in connectivity patterns between regions (Baums et al. 2006). Because of the heterogeneity of the coral reef habitat, the patterns of connectivity may also change with the scales of the seascape layer in the model. This aspect, together with the effect of integration time steps, requires further investigation. To accurately estimate population connectivity and local retention with online models, the seascape data need to be coupled with Lagrangian tracking.

Although oceanographic distances measured in total displacement show similar precision between all LSM configurations presented here, analyses using transition matrices reveal that both the dispersal distances from the source populations and the spatial arrangement of the connections (i.e. end points of the trajectories) may differ between models and are very sensitive to the mesoscale variability typical to the flow dynamics 
in coastal regions. As a result, the length of the path is similar and related to the integration time and the mean current, but the paths are different with the various models, which is of critical interest in connectivity studies. Indeed, offline-tracking models in which $T_{\mathrm{L}}$ and $\sigma$ are constant over the entire domain cannot predict the end point of long-term trajectories. We selected a region with complex geomorphology and circulation (Ezer et al. 2005, Tang et al. 2006) and demonstrated that LSMs without spin have the lowest accuracy there. Consequently, spin is an important parameter to take into account when modeling larval transport in coastal areas, particularly in coral reef ecosystems.

In summary, the accuracy of offline models depends considerably on their parameterization, and it appears that models with spatially explicit values for the Lagrangian decorrelation time scale, $T_{\mathrm{L}}$, and the variance of the velocity field, $\sigma$, offer potentially increased accuracy. This work underscores the relative roles of biological and physical processes in patterns of larval exchange and demonstrates the need for careful parameterization. Further validation with field studies is also vital. We have shown that the impacts of larval behavior extend beyond enhancing the process of selfrecruitment by changing population connectivity patterns as much as eddies do. Finally, the consequences of vertical migration and survival emerge as key components in population connectivity estimates and need to be further coupled.

Acknowledgements. We thank Elizabeth North, Alejandro Gallego, and Pierre Petitgas, co-chairs of the Workshop on 'Advancements in Modelling Physical-Biological Interactions in Fish Early-Life History: Recommended Practices and Future Directions (WKAMF)', and the anonymous reviewers who helped in focusing this contribution. We are also grateful to Ashwanth Srinivasan for help with the coupled-model algorithm, and to Cedric Guigand for the photos of fish larvae. This work was supported by a joint World Resources Institute and The Nature Conservancy grant to C.B.P. and L.M.C. L.M.C. was also supported by National Science Foundation Grant OCE 03-271808. Partial funding for this work was provided by the Coral Reef Targeted Research (CRTR) Program Connectivity Working Group, a program of the Global Environment Facility (GEF), the World Bank, The University of Queensland (Australia), and the United States National Oceanic and Atmospheric Administration (NOAA).

\section{LITERATURE CITED}

Barber PH, Palumbi SR, Erdmann MV, Moosa MK (2002) Sharp genetic breaks among populations of a benthic marine crustacean indicate limited oceanic larval transport: patterns, cause, and consequence. Mol Ecol 11:659-674

Baums I, Paris CB, Cherubin LM (2006) A bio-oceanographic filter to larval dispersal in a reef-building coral. Limnol Oceanogr 51(5):1969-1981

Berloff PS, McWilliams JC (2002) Material transport in oceanic gyres. Part II: Hierarchy of stochastic models. J Phys Oceanogr 32:797-830
Berloff PS, McWilliams JM (2003) Material transport in oceanic gyres. Part III: Randomized stochastic models. J Phys Oceanogr 33:1416-1445

Blayo E, Debreu L (1999) Adaptive mesh refinement for finite-difference ocean models: first experiments. J Phys Oceanogr 29:1239-1250

Borgas MS, Flesch TK, Sawford BL (1997) Turbulent dispersion with broken reflexional symmetry. J Fluid Mech 332: 25-54

Burke L, Maidens J (eds) (2004) Reefs at risk in the Caribbean. World Resources Institute, Washington, DC

Chérubin LM, Kuchinke C, Paris CB (2007) Ocean circulation and terrestrial runoff dynamics in the meso-American region. Coral Reefs (in press)

Codling EA, Hill NA, Pitchford JW, Simpson SD (2004) Random walk models for the movement and recruitment of reef fish larvae. Mar Ecol Prog Ser 279:215-224

Cowen (2002) Oceanographic influences on larval dispersal and retention and their consequences for population connectivity. In: Sale P (ed) Coral reef fishes. Academic Press, London, p 149-170

Cowen RK, Lwiza KMM, Sponaugle S, Paris CB, Olson DB (2000) Connectivity of marine populations: open or closed? Science 287:857-859

Cowen RK, Paris CB, Srinivasan A (2006) Scaling connectivity in marine populations. Science 311:522-527

deYoung B, Heath M, Werner F, Chai F, Megrey B, Monfray P (2004) Challenges of modeling ocean basin ecosystems. Science 304:1463-1466

Ezer T, Thattai DV, Kjerfve B, Heyman WD (2005) On the variability of the flow along the meso-American barrier reef system: a numerical model study of the influence of the Caribbean current and eddies. Ocean Dyn 55(5-6): 458-475

Fiksen Ø, Jørgensen C, Kristiansen T, Vikebø F, Huse G (2007) Linking behavioural ecology and oceanography: larval behaviour determines growth, mortality and dispersal. Mar Ecol Prog Ser 347:195-205

Fisher R, Bellwood DR (2002) The influence of swimming speed on sustained swimming performance of late-stage reef fish larvae. Mar Biol 140:801-807

Fisher R, Bellwood DR, Job SD (2000) Development of swimming abilities in reef fish larvae. Mar Ecol Prog Ser 202: 163-173

Gerlach G, Atema J, Kingsford MJ, Black KP, Miller-Sims V (2007) Smelling home can prevent dispersal of reef fish larvae. Proc Nat Acad Sci USA 104:858-863

Griffa A (1996) Applications of stochastic particle models to oceanographic problems. In: Adler R, Muller P, Rozovoskii B (eds) Stochastic modeling in physical oceanography. Birkhäuser, Basel, p 114-140

Hanski I, Gaggiotti OE (2004) Ecology, genetics, and evolution of metapopulation. Elsevier, Burlington, MA

Hasting A, Botsford LW (2006) Persistence of spatial populations depends on returning home. Proc Natl Acad Sci USA 103(15):6067-6072

Hedrick (2000) Genetics of populations, 2nd edn. Jones \& Bartlett Publishers, Sudbury, MA

Hermann AJ, Hinckley S, Megrey BA, Napp JM (2001) Applied and theoretical considerations for constructing spatially explicit individual-based models of marine fish early life history which include multiple trophic levels. ICES J Mar Sci 58:1030-1041

Houde E (1989) Comparative growth, mortality, and energentics of marine fish larvae - temperature and implied latitudinal effects. Fish Bull 87(3):471-495

James MK, Armsworth PR, Mason LB, Bode L (2002) The structure of reef fish metapopulations: modelling larval 
dispersal and retention patterns. Proc R Soc Lond B (269): 2079-2086

Jones GP, Planes S, Thorrold SR (2005) Coral reef fish larvae settle close to home. Curr Biol 15:1314-1318

Kinlan BP, Gaines SD, Lester SE (2005) Propagule dispersal and the scales of marine community process. Diversity Distrib 11:139-148

Kobayashi DR (2006) Colonization of Hawaiian Archipelago via Johnston Atoll: a characterization of oceanographic transport corridors for pelagic larvae using computer simulation. Coral Reefs 25(3):407-417

Leis JM (2006) Are larvae of demersal fishes plankton or nekton? Adv Mar Biol 51:59-141

Leis JM (2007) Behaviour as input for modelling dispersal of fish larvae: behaviour, biogeography, hydrodynamics, ontogeny, physiology and phylogeny meet hydrography. Mar Ecol Prog Ser 347:185-193

Levin LA (2006) Recent progress in understanding larval dispersal: new directions and digressions. Integr Comp Biol 46(3):282-297

Levins R (1969) Some demographic and genetic consequences of environmental heterogeneity for biological control. Bull Entomol Soc Am 15:237-240

Lindeman KC, Richards WJ, Lyczkowski-Shultz J, Drass DM, Paris CB, Leis JM, Lara M, Comyns BH (2005) Lutjanidae: snappers. In: Richards WJ (ed) Early stages of Atlantic fishes. CRC Press, Boca Raton, FL, p 1549-1586

Lumpkin R, Treguier AM, Speer K (2002) Lagrangian eddy scales in the northern Atlantic Ocean. J Phys Oceanogr 32: $2425-2440$

Marchesiello P, Mcwilliams JC, Shchepetkin A (2003) Equilibrium structure and dynamics of the California Current System. J Phys Oceanogr 33:753-783

Middleton J (1985) Drifter spectra and diffusivities. J Mar Res 43:37-55

Okubo A (1971) Oceanic diffusion diagrams. Deep-Sea Res 18: 789-802

Paris CB, Cowen RK (2004) Direct evidence of a biophysical retention mechanism for coral reef fish larvae. Limnol Oceanogr 49(6):1964-1979

Paris CB, Cowen RK, Lwiza KMM, Wang DP, Olson DB (2002) Objective analysis of three-dimensional circulation in the vicinity of Barbados, West Indies: implication for larval transport. Deep-Sea Res 49:1363-1386

Paris CB, Sponaugle, S, Cowen RK, Rotunno T (2005a) Pomacentridae: damselfishes. In: Richards JW (ed) Early stages of Atlantic fishes. CRC Press, Boca Raton, FL, p 1787-1818

Paris CB, Cowen RK, Claro R, Lindeman KC (2005b) Larval transport pathways from Cuban snapper (Lutjanidae) spawning aggregations based on biophysical modeling. Mar Ecol Prog Ser 296:93-106

Paris-Limouzy CB (2001) Transport dynamics and survival of the pelagic larval stages of a coral reef fish, the bicolor damselfish, Stegastes partitus (Poey). PhD thesis, Marine

Editorial responsibility: Alejandro Gallego (Contributing Editor), Aberdeen, UK
Sciences Research Center, State University of New York, Stony Brook, NY

Reynolds AM (2002a) Lagrangian stochastic modeling of anomalous diffusion in two-dimensional turbulence. Phys Fluids 14:1442-144

Reynolds AM (2002b) On Lagrangian stochastic modeling of material transport in oceanic gyres. Physica D 172: $124-138$

Richardson PL (2005) Caribbean Current and eddies as observed by surface drifters. Deep-Sea Res II 52:429-463

Sawford BL (1999) Rotation of trajectories in Lagrangian stochastic models of turbulent dispersion. Boundary-Layer Meteorol 93:411-424

Shchepetkin A, McWilliams JC (2004) The regional oceanic modeling system: a split-explicit, free-surface, topographyfollowing-coordinate ocean model. Ocean Model 9: 347-404

Siegel DA, Kinlan BP, Gaylord B, Gaines SD (2003) Lagrangian descriptions of marine dispersion. Mar Ecol Prog Ser 260:83-96

Steneck RS (2006) Staying connected in a turbulent world. Science 311:480-481

Steneck RS, Cowen RK, Paris CB, Srinivasan A (2006) Connectivity in marine protected areas-response. Science 313: $44-45$

Tang L, Sheng J, Hatcher BG, Sale PF (2006) Numerical study of circulation. Dispersion, and hydrodynamic connectivity of surface waters on the Belize shelf. J Geophys Res 111: C01003. doi:10.1029/2005JC002930

Thomson D (1987) Criteria for the selection of stochastic models of particle trajectories in turbulent flows. J Fluid Mech 180:529-556

Thorrold SR, Jones GP, Hellberg ME, Burton RS, Swearer SE, Neigel JE, Morgan SG, Warner RR (2002) Quantifying larval retention and connectivity in marine populations with artificial and natural markers. Bull Mar Sci 70:291-308

Urban D, Keitt T (2001) Landscape connectivity: a graphtheoretic perspective. Ecology 82:1205-1218

Veneziani M, Griffa A, Reynolds AM, Mariano AJ (2004) Oceanic turbulence and stochastic models from subsurface Lagrangian data for the Northwest Atlantic Ocean. J Phys Oceanogr 34(8):1884-1906

Veneziani M, Griffa A, Garraffo ZD, Chassignet EP (2005a) Lagrangian spin parameter and coherent structures from trajectories in a high resolution model. J Mar Res 63: 753-788

Veneziani M, Griffa A, Reynolds AM, Garraffo ZD, Chassignet EP (2005b) Parameterization of Lagrangian spin statistics and particle dispersion in the presence of coherent vortices. J Mar Res 63:1057-1083

Werner FE, Quinlan JA, Lough RG, Lynch DR (2001) Spatially-explicit individual based modeling of marine populations: a review of the advances in the 1990s. Sarsia 86: $411-421$

Submitted: August 9, 2006; Accepted: August 31, 2007

Proofs received from author(s): September 25, 2007 